\title{
Specific inhibition of Streptococcus bovis by endolysin LyJH307 supplementation shifts the rumen microbiota and metabolic pathways related to carbohydrate metabolism
}

\section{Han Been KIM}

Pusan National University - Milyang Campus https://orcid.org/0000-0003-2803-7318

\section{Tansol Park}

Chungang University - Ansan Campus: Chung Ang University - Anseong Campus

Inhyuk Kwon

EASY BIO, Inc.

Jakyeom Seo ( $\nabla$ jseo81@pusan.ac.kr)

https://orcid.org/0000-0002-9176-5206

\section{Research}

Keywords: Endolysin, Streptococcus bovis, Ruminal acidosis, Rumen microbiota

Posted Date: March 5th, 2021

DOI: https://doi.org/10.21203/rs.3.rs-269499/v1

License: () (i) This work is licensed under a Creative Commons Attribution 4.0 International License.

Read Full License

Version of Record: A version of this preprint was published at Journal of Animal Science and Biotechnology on August 4th, 2021. See the published version at https://doi.org/10.1186/s40104-02100614-x. 


\section{Abstract}

Background: Ruminal acidosis negatively affects the animal production and performances of highperformance cattle by abnormal balancing of the rumen microbiota, which caused significant economic damage to farmers. Endolysins, the bacteriophage-originated peptidoglycan hydrolases, are a promising replacement of antibiotics due to their immediate lytic activity and no evidence of antibiotic resistance. The objectives of this study were to investigate the lytic activity of Streptococcus bovis specific endolysin LyJH307 against $S$. bovis and explore changes in rumen fermentation and microbiota in an in vitro system. Treatments consisted of corn grain with protein elution buffer and corn grain with endolysin LyJH307 (LyJH307, $0.2 \%$ of dietary dry matter). The rumen bacterial community was analyzed using $16 \mathrm{~S}$ rRNA amplicon sequencing, followed by the prediction of microbial functional features by using PICRUSt2.

Results: Endolysin LyJH307 supplementation at $6 \mathrm{~h}$ incubation time markedly decreased the absolute abundance of S. bovis (approximately $70 \%$ ) and increased ruminal $\mathrm{pH}$ at the end of the incubation. There were no significant changes in gas production, in vitro dry matter degradability, and total volatile fatty acid production. The acetate proportion was significantly increased after LyJH307 addition, whereas propionate was decreased, thereby inducing an increase of acetate to propionate ratio in LyJH307. LyJH307 supplementation increased D-lactate without any change in L-lactate concentration. Among the alpha diversity indices, there were no significant differences in Shannon's index, Simpson's index, Chao1 estimates, and evenness. Based on Bray-Curtis dissimilarity matrices, the LyJH307 affected the overall shift in ruminal microbiota. Endolysin LyJH307 supplementation induced an increase of 11 genera containing Lachnoclostridium, WCHB1-41, unclassified genus Selenomonadaceae, Paraprevotella, vadinBE97, Ruminococcus gauvreauii group, Lactobacillus, Anaerorhabdus furcosa group, Victivallaceae, Desulfuromonadaceae, and Sediminispirochaeta. The predicted functional features represented by the Kyoto Encyclopedia of Genes and Genomes pathways were changed by LyJH307 toward a decrease of carbohydrate metabolism.

Conclusions: Endolysin LyJH307 caused a reduction of S. bovis and an increase of ruminal pH with concomitant shifts in minor rumen microbiota and its metabolic pathways related to carbohydrate metabolism. This study provides the first insight into the availability of endolysin as a specific modulator for rumen and shows the possibility of endolysin degradation by rumen microbiota.

\section{Background}

The use of high amounts of non-fiber carbohydrate (NFC) is generally accepted by farmers to meet the nutritional energy requirements of high-performance cattle; however, this strategy often carries nutritional disorders, including the occurrence of ruminal acidosis. Generally, ruminal acidosis can be divided into two types, subacute ruminal acidosis (SARA) and acute acidosis [1]. The outbreak of ruminal acidosis starts from increased inflow of NFC into the rumen, and it can stimulate the overgrowth of amylolytic bacteria, which induce the increase of volatile fatty acids (VFA) and lactate production and decrease 
ruminal $\mathrm{pH}$ [2]. In this condition, excessive production of VFA with a concomitant increase of lactate fermenting bacteria produces SARA; however, improper growth of lactate fermenting bacteria can induce acute acidosis by the accumulation of lactate in the rumen $[1,3]$. Both types of acidosis can cause a decrease in production performances (average daily gain, growth rate, and milk yield) and create an abnormal balance of rumen microbiota, causing significant economic damage to farmers.

Streptococcus bovis is one of the main ruminal amylolytic bacteria that can produce lactate as the major fermentation product under low ruminal $\mathrm{pH}$ conditions. Under a high grain diet, $S$. bovis has characteristics that generate more ATP per hour than other ruminal amylolytic bacteria, high acid resistance, and rapid growth rate $[4,5]$. Therefore, $S$. bovis is one of the critical initiators of ruminal acidosis.

Great efforts have been undertaken to prevent an outbreak of ruminal acidosis, such as a change of feeding strategies (e.g., a gradual increase of concentration source) [6], use of buffering agent [7], or feeding rumen modulators such as chemical agent (a-amylase and glucosidase inhibitor) [8]. However, to the best of our knowledge, there have been no studies attempting to prevent rumen acidosis by the specific inhibition of $S$. bovis in an in vitro rumen model.

Endolysin is a bacteriophage-originated peptidoglycan (PG) hydrolases that can break down the PG layer of the host from within the bacteria during the final stage of the replication cycle [9]. In general, endolysin targeting gram-positive bacteria comprises at least two conserved functional domains, $\mathrm{N}$-terminal enzymatically active domain (EAD) and C-terminal cell wall binding domain (CBD). The CBD affects host specificity by recognizing and binding to the host PG layer, and the EAD can degrade the PG layer when increased endolysin-PG proximity is made by CBD [10]. The number of studies related to endolysin development has been improved by the emergence of multi-drug resistant bacteria, which render many types of antibiotics ineffective. Many researchers have shown that endolysin can lyse gram-positive bacteria when endolysin is administered externally because of the absence of an outer membrane such as a lipopolysaccharide layer [11-13]. Therefore, endolysin is a promising replacement for antibiotics. In the food and biomedical health field, many endolysin applications have tried to solve the problem of antibiotic resistance [14]; however, in the livestock science field, to the best of our knowledge, limited trials have been reported on endolysin development for treating mastitis-associated bacteria, specifically Staphylococcus aureus [15], and infectious bacteria on fresh cheese, specifically Listeria monocytogenes [16]. Moreover, there have been no attempts to apply endolysin for modulating ruminal metabolic disease via the specific control of ruminal bacteria in the rumen.

In a previous study, we developed endolysin LyJH307 with high lytic activity against $S$. bovis [11]. Endolysin LyJH307 has a distinct combination of conserved domain consisting of N-terminal NlpC/P60 superfamily (EAD) and C-terminal Zoocin A target recognition domain superfamily (CBD), and the endolysin Ly JH307 had potent lytic activity in a variety of condition $\left(\mathrm{pH}, 5.0-6.0\right.$; temperature, $25-55^{\circ} \mathrm{C}$; $\mathrm{NaCl}$ concentration, 0-250 mM). Moreover, it confirmed that endolysin LyJH307 retained lytic activity 
against the $S$. bovis strain isolated from the rumen. Therefore, we expected that endolysin LyJH307 would have potent lytic activity against the $S$. bovis group in rumen condition.

Therefore, the objectives of the present study were to (1) investigate the lytic activity of endolysin LyJH307 against $S$. bovis in an in vitro system, and (2) explore the changes in rumen fermentation and microbiota.

\section{Methods}

The protocols for this study concerning animal use were reviewed and approved by the Animal Research Ethics Committee of Pusan National University (Pusan, Korea, PNU-2020-2827).

\subsection{Preparation of recombinant endolysin LyJH307}

Recombinant LyJH307 was cloned in Escherichia coli BL21 (DE3), and the effect of lytic activity on the $S$. bovis group was tested following the methodology described by Kim, Lee, Kwon (11). To produce the recombinant endolysin LyJH307, the transformant was grown in Luria-Bertani medium (Difco Laboratories Inc.) until the optical density at $600 \mathrm{~nm}\left(\mathrm{OD}_{600 \mathrm{~nm}}\right)$ reached 0.4 . After that, $1 \mathrm{mM}$ isopropyl- $\beta$ D-thiogalactoside was added to the medium, and the cells were further incubated for $4 \mathrm{~h}$ at $37^{\circ} \mathrm{C}$. Harvested cells were suspended in lysis buffer $\left(50 \mathrm{mM} \mathrm{NaH}_{2} \mathrm{PO}_{4}, 300 \mathrm{mM} \mathrm{NaCl}\right.$, and $10 \mathrm{mM}$ imidazole at pH 8.0), and lysed by sonication (KYY-80, Korea Process Technology Co., Ltd., Seoul, Korea). After centrifugation at $10,000 \times g$ for 15 min, the supernatant was passed through Ni-NTA Agarose (Qiagen $\mathrm{GmbH}$, Hilden, Germany) and the recombinant LyJH307 was purified as described by the manufacturer, and resolved by sodium dodecyl sulfate-polyacrylamide gel electrophoresis. The purified endolysin was incubated with $5 \mathrm{mM}$ ethylenediaminetetraacetic acid at $25^{\circ} \mathrm{C}$ for $30 \mathrm{~min}$ to chelate divalent cations attached to the endolysin. The ethylenediaminetetraacetic acid-treated LyJH307 was concentrated with an Amicon Ultra-4 (10 kDa, Merck KGaA, Darmstadt, Germany), and the purified LyJH307 was mixed with elution buffer ( $50 \mathrm{mM} \mathrm{NaH}_{2} \mathrm{PO}_{4}, 300 \mathrm{mM} \mathrm{NaCl}$, and $10 \mathrm{mM} \mathrm{CaCl}_{2}$ at pH 8.0).

\subsection{Experimental diets and chemical analysis}

To induce the acidosis condition within $12 \mathrm{~h}$ under in vitro fermentation, we selected corn grain as the experimental diet. The chemical composition of the corn was as follows: dry matter (DM), 88.5\%; crude protein (CP), 8.4\%DM; neutral detergent fiber (aNDF), 12.4\%DM; acid detergent fiber (ADF), 5.3\%DM; ether extract (EE), 4.7\%DM; starch, 70.6\%DM; sugar, 3.4\%DM; non-fibrous carbohydrate, 74.0\%DM; ash, 1.1\%DM; and lignin, 1.7\%DM. Before chemical analysis was conducted, the corn grain was dried at $60^{\circ} \mathrm{C}$ for $96 \mathrm{~h}$ and ground with a cyclone mill (Foss Tecator Cyclotec 1093, Foss, Hillerød, Denmark) fitted with a $1 \mathrm{~mm}$ screen. The DM (\#934.01), CP (\#990.03), ADF (\#973.18), and ash (\#942.05) were analyzed by AOAC international methods [17]. The EE (\#2003.05) was assessed using AOAC international methods [18]. The $\mathrm{CP}$ was calculated by multiplying the nitrogen content by 6.25 , and total nitrogen was measured by the Kjeldahl method with a nitrogen combustion analyzer (Leco FP-528 Leco, MI, USA). The aNDF and lignin were analyzed [19] to determine the fiber content. Heat-stable amylase (a-amylase) was used to 
estimate aNDF and was expressed inclusive of residual ash. The NFC of the experimental diet was estimated as follows: NFC $=[100-$ ash $-\mathrm{EE}-\mathrm{CP}-$ aNDF $]$.

\subsection{Experimental treatment}

A complete randomized block design was used for the experiment, with treatment as the main effect. Two experimental treatments were used as follows: (1) Control diet (CON) containing corn grain with elution buffer, and (2) endolysin LyJH307 treatment (LyJH307) containing corn grain with $0.2 \% \mathrm{DM}$ of endolysin LyJH307. The volume of elution buffer supplemented was the same as that of the endolysin in the LyJH307 treatment.

\subsection{In vitro rumen fermentation}

In vitro fermentation was undertaken using the rumen fluid collected from two cannulated Holsteins (body weight $=450 \pm 30 \mathrm{~kg}$ ) before the morning feed at the Center for Agriculture Research, Pusan National University, Korea. Animals were fed a diet of $600 \mathrm{~g} / \mathrm{kg}$ Timothy hay and $400 \mathrm{~g} / \mathrm{kg}$ of a commercial concentrate mix. The rumen fluid was collected before the morning feeding time, mixed, transferred into a thermos bottle, and immediately transported to the laboratory. The rumen contents were filtered through eight layers of cheesecloth and mixed with $3 \times$ volumes of in vitro rumen buffer solution [20]. Approximately $1 \mathrm{~g}$ of the ground experimental substrate was placed into pre-weighed nylon bags (R510, Ankom Technology, NY, USA). All bags were heat-sealed and transferred into empty $50 \mathrm{~mL}$ serum bottles. Three bottles were used per dietary treatment. Then, $20 \mathrm{~mL}$ of the mixture of rumen fluid and buffer was transferred, accompanied by continuous flushing with $\mathrm{O}_{2}$-free $\mathrm{CO}_{2}$ gas. The bottles were sealed with butyl rubber stoppers and aluminum caps and were incubated on a rotary shaker (JSSI-300T, JS Research Inc., Gongju, Korea) at $20 \mathrm{rpm}$ for $12 \mathrm{~h}$ at $39^{\circ} \mathrm{C}$. After incubation for $6 \mathrm{~h}$, the gas production was measured, and elution buffer and $\mathrm{LyJH} 307$ solution pre-flushed with $\mathrm{O}_{2}$-free $\mathrm{CO}_{2}$ gas were injected into each treatment, and the remaining gas was removed. Gas production was measured at 6,9 , and $12 \mathrm{~h}$ using a pressure transducer (Sun Bee Instrument Inc., Seoul, Korea) as described by Theodorou, Williams, Dhanoa, McAllan and France (21).

After measuring the final gas production, the bottle caps were removed, and the bottles were fixed immediately on ice to stop the fermentation. The nylon bags were then removed from the bottles and rinsed under flowing water until the rinsed water ran clear. The washed bags were dried at $60^{\circ} \mathrm{C}$ for $72 \mathrm{~h}$ and weighed to measure the in vitro DM digestibility (IVDMD). Sample fluid $(5 \mathrm{~mL})$ was centrifuged at $20,000 \times g$ for $20 \mathrm{~min}$ at $4^{\circ} \mathrm{C}$, the supernatant was discarded, and the pellet was stored at $-80^{\circ} \mathrm{C}$ until rumen microbial population analysis. The remaining culture fluid was then transferred to a centrifugal tube for centrifugation at $3,500 \mathrm{rpm}$ and $4^{\circ} \mathrm{C}$ for $20 \mathrm{~min}$. The supernatant was collected for the determination of $\mathrm{pH}$ and VFA and ammonia-nitrogen $\left(\mathrm{NH}_{3}-\mathrm{N}\right)$ concentration. The $\mathrm{pH}$ of the culture fluid was measured with a pH meter (FP20, Mettler Toledo, OH, USA). The supernatant for the VFA analysis was acidified with $200 \mu \mathrm{L}$ of $25 \%$ meta-phosphoric acid, and the supernatant for the $\mathrm{NH}_{3}-\mathrm{N}$ analysis was 
acidified with $200 \mu \mathrm{L}$ of $0.2 \mathrm{M}$ sulfuric acid, and both were stored at $-20^{\circ} \mathrm{C}$ until subsequent VFA and $\mathrm{NH}_{3}{ }^{-}$ $\mathrm{N}$ analyses.

For VFA analysis, $200 \mu \mathrm{L}$ of the supernatant was diluted with $800 \mu \mathrm{L}$ of ethyl alcohol anhydrous (40232304, Daejung Chemicals, Siheung, Korea) after $20 \mathrm{~min}$ of centrifugation at 20,000 $\times g$. VFA was measured with gas chromatography (Agilent 7890A, Agilent Technology, CA, USA) equipped with a flame ionization detector and capillary column (Nukol ${ }^{\mathrm{TM}}$ Fused silica capillary column, $30 \mathrm{~m} \times 250 \mu \mathrm{m} \times 0.25$ $\mu \mathrm{m}$, Supelco Inc., PA, USA). The temperature of the oven, injector, and detector was set at $90^{\circ} \mathrm{C}$, $90-200^{\circ} \mathrm{C}$, and $230^{\circ} \mathrm{C}$, respectively. Nitrogen was used as the carrier gas at a flow rate of $30 \mathrm{~mL} / \mathrm{min}$. The $\mathrm{NH}_{3}-\mathrm{N}$ concentration was analyzed with several modifications [22]. A $2 \mu \mathrm{L}$ sample of the supernatant was mixed with $100 \mu \mathrm{L}$ of phenol color reagent $(50 \mathrm{~g}$ of phenol, $0.25 \mathrm{~g}$ of sodium nitroferricyanide, and $1 \mathrm{~L}$ of distilled water) and alkali hypochlorite $(25 \mathrm{~g}$ of sodium hydroxide, $16.8 \mathrm{~mL}$ of sodium hydroxide, and $1 \mathrm{~L}$ of distilled water) after the samples were centrifuged at $20,000 \times g$ for $20 \mathrm{~min}$ at $4^{\circ} \mathrm{C}$. The mixture was then incubated in a water bath at $37^{\circ} \mathrm{C}$ for $15 \mathrm{~min}$. $\mathrm{The}^{\mathrm{NH}_{3}}-\mathrm{N}$ concentration was determined by measuring the optical density at $630 \mathrm{~nm}$ by using a microplate reader (iMARK, Bio-Rad Laboratories, Inc., CA, USA).

\subsection{DNA extraction}

Total DNA was extracted from the pellet stored at $-80^{\circ} \mathrm{C}$ by using the repeated bead beating plus column $(\mathrm{RBB}+\mathrm{C})$ method [23]. Genomic DNA was treated with RNase $\mathrm{A}$ and proteinase $\mathrm{K}$ and purified using columns from the DokDo-Prep Genomic DNA Kit (Elpis-Biotech, Daejeon, Korea). The total DNA concentration and purity were measured using a NanoDrop (ND-1000, Thermo Fisher, MA, USA). The purified DNA was stored at $-20^{\circ} \mathrm{C}$ until amplicon sequencing and real-time polymerase chain reaction (PCR).

\subsection{Quantitative real-time $P C R$}

To assess the absolute abundance of rumen microbes in each sample, quantitative real-time PCR assays were performed on a CFX 96 Touch system (Bio-Rad Laboratories, Inc.) by using several primer sets described in Table 1, as performed in our previous studies [24].

Table 1. PCR primers used in quantitative real-time PCR 


\begin{tabular}{|c|c|c|c|c|c|}
\hline $\begin{array}{l}\text { Target } \\
\text { species }\end{array}$ & Primer & Sequence $\left(5^{\prime} \rightarrow 3^{\prime}\right)$ & $\begin{array}{l}\text { Size } \\
\text { (bp) }\end{array}$ & Efficiency ${ }^{1)}$ & References \\
\hline \multirow{2}{*}{$\begin{array}{l}\text { General } \\
\text { bacteria }\end{array}$} & $\mathrm{F}$ & CGGCAACGAGCGCAACCC & \multirow[t]{2}{*}{130} & \multirow[t]{2}{*}{1.975} & \multirow[t]{2}{*}{ [66] } \\
\hline & $\mathrm{R}$ & CCATTGTAGCACGTGTGTAGCC & & & \\
\hline \multirow{3}{*}{$\begin{array}{l}\text { Ciliate } \\
\text { protozoa }\end{array}$} & $\mathrm{F}$ & GCTTTCGWTGGTAGTGTATT & \multirow[t]{3}{*}{223} & \multirow[t]{3}{*}{1.872} & \multirow[t]{3}{*}{ [67] } \\
\hline & $\mathrm{R}$ & CTTGCCCTCYAATCGTWCT & & & \\
\hline & $\mathrm{R}$ & CCCATCCTATAGCGGTAAACCTTTG & & & \\
\hline \multirow{2}{*}{$\begin{array}{l}\text { Streptococcus } \\
\text { bovis }\end{array}$} & $\mathrm{F}$ & TTCCTAGAGATAGGAAGTTTCTTCGG & \multirow[t]{2}{*}{127} & \multirow[t]{2}{*}{1.944} & \multirow[t]{2}{*}{ [68] } \\
\hline & $\mathrm{R}$ & ATGATGGCAACTAACAATAGGGGT & & & \\
\hline
\end{tabular}

bp, base pair.

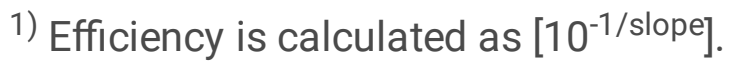

\subsection{PCR amplification and sequencing}

PCR amplification was performed using fusion primers targeting the V3 to V4 regions of the 16S rRNA gene with the extracted DNA. For bacterial amplification, the fusion primers used were $341 \mathrm{~F}\left(5^{\prime}\right.$ AATGATACGGCGACCACCGAGATCTACAC-XXXXXXXX-TCGTCGGCAGCGTC-AGATGTGTATAAGAGACAGCCTACGGGNGGCWGCAG-3'; underlined sequence indicates the target region primer) and 805R (5'CAAGCAGAAGACGGCATACGAGAT-XXXXXXXX-GTCTCGTGGGCTCGG-AGATGTGTATAAGAGACAGGACTACHVGGGTATCTAATCC-3'). The fusion primers were constructed in the following order: P5 (P7) graft binding, i5 (i7) index, Nextera consensus, sequencing adaptor, and target region sequence. The amplifications were performed under the following conditions: initial denaturation at $95^{\circ} \mathrm{C}$ for $3 \mathrm{~min}$, followed by 25 cycles of denaturation at $95^{\circ} \mathrm{C}$ for $30 \mathrm{~s}$; primer annealing at $55^{\circ} \mathrm{C}$ for $30 \mathrm{~s}$; and extension at $72{ }^{\circ} \mathrm{C}$ for $30 \mathrm{~s}$, with a final elongation at $72{ }^{\circ} \mathrm{C}$ for $5 \mathrm{~min}$. The PCR product was confirmed using $1 \%$ agarose gel electrophoresis and visualized under a Gel Doc system (Bio-Rad). The amplified products were purified with CleanPCR (CleanNA). Equal concentrations of purified products were pooled together, and short fragments (non-target products) were removed with CleanPCR (CleanNA). The quality and product size were assessed on a Bioanalyzer 2100 (Agilent, Palo Alto, CA, USA) using a DNA 7500 chip. Mixed amplicons were pooled and the sequencing was undertaken at Chunlab, Inc. (Seoul, Korea), with an Illumina MiSeq Sequencing System (Illumina, USA) according to the manufacturer's instructions.

\subsection{Data analysis pipeline}

To analyze the 16S rRNA gene amplicon sequences, the sequencing data were analyzed using Quantitative Insights into Microbial Ecology 2 (QIIME2) [25]. Briefly, after demultiplexing, the primer sequences were removed using FASTX-Toolkit [26]. The sequence reads were quality-filtered, denoized, and the paired-end sequence data were merged using FLASH2 [27]. The chimeric sequences were then 
removed using the DADA2 plugin, and amplicon sequencing variants (ASVs) were taxonomically classified from the Silva (SSU138) 16S rRNA gene database [28]. Sequences classified as mitochondria, chloroplast, and unassigned ASVs were removed from the dataset. The alpha diversities (Shannon's index, Simpson's index, Chao1 estimates, and evenness) were calculated from rarefied abundance tables using 14,673 sequences per sample. To investigate the dissimilarity of overall microbiota between treatments, principal coordinate analysis (PCoA) based on Bray-Curtis dissimilarity and weighted UniFrac distance matrices were used. The functional metagenomic predictions were generated using a PICRUSt2 algorithm (version 2.3.0-b) [29].

\subsection{Statistical analysis}

All data were checked for normal distribution by using the Shapiro-Wilk test in SAS 9.4 (SAS Institute Inc., NC, USA). Normally distributed data including all alpha diversity measurements (Shannon's index, Simpson's index, Chao1 estimates, and evenness), rumen fermentation data (gas production, IVDMD, $\mathrm{NH}_{3}-\mathrm{N}, \mathrm{VFA}$, and lactate), and various functional features predicted from the 16S rRNA gene using five different reference databases (KEGG, Pfam, clusters of orthologous genes [COG], enzyme classification, and MetaCyc) were further analyzed using t-tests in SAS 9.4. Statistical significance for normally distributed data was declared at $P<0.05$, and a trend was speculated at $0.05 \leq P \leq 0.10$. The PCoA results of beta diversity were analyzed by permutational multivariate analysis of variance (PERMANOVA) to assess if microbial community significantly differed between treatments. A major microbiome was defined as those ASVs present in greater than $50 \%$ of the samples. The differences in major microbiome and functional features (KEGG pathway and module) were analyzed by the linear discriminant analysis effect size (LEfSe), which implements a non-parametric Kruskal-Wallis sum-rank test followed by linear discriminate analysis. Spearman's rank correlations between the relative abundance of differentially abundant prokaryotic taxa revealed by LEfSe and fermentation data were analyzed using PROC CORR procedures of SAS 9.4 and visualized using the R package corrplot (v. 3.6.3).

\section{Results}

\subsection{Effect of endolysin LyJH307 on Ruminal fermentation characteristics and qPCR}

Compared to $\mathrm{CON}$, endolysin Ly JH307 supplementation did not affect gas production in any of the investigated incubation times (Table 2). The pH was significantly higher in the Ly JH307 group than in the CON group $(P=0.0335)$, whereas IVDMD, $\mathrm{NH}_{3}-\mathrm{N}$, and total VFA production were not changed by endolysin LyJH307 supplementation (IVDMD, $P=0.3597 ; \mathrm{NH}_{3}-\mathrm{N}, P=0.1485$; and total VFA, $P=0.2592$ ). In each proportion of individual VFAs, the proportion of acetate was significantly higher in the Ly JH307 group than in the CON group $(P=0.0362)$, whereas that of propionate was significantly lower in the Ly JH307 group $(P=0.0379)$, thereby increasing the acetate to propionate ratio (A:P ratio) in LyJH307 $(P=0.0291)$. However, endolysin LyJH307 supplementation did not affect the proportion of butyrate, iso-butyrate, valerate, and iso-valerate (butyrate, $P=0.2435$; iso-butyrate, $P=0.3502$; valerate, $P=0.2752$; and isovalerate, $P=0.3502$ ). Endolysin Ly JH307 supplementation significantly increased only the D-lactate 
concentration compared to the CON group $(P=0.0340)$ and total lactate concentration was high in the LyJH307 group $(P=0.0824)$.

Table 2. Gas production and rumen fermentation parameters by endolysin LyJH307 supplementation in an in vitro experiment at $12 \mathrm{~h}$ of incubation

\begin{tabular}{|c|c|c|c|c|}
\hline \multicolumn{5}{|c|}{ Treatments ${ }^{1}$ ) } \\
\hline Items ${ }^{*}$ & $\mathrm{CON}$ & LyJH307 & SEM & $P$-value \\
\hline Gas $6 \mathrm{~h}, \mathrm{~mL} / \mathrm{g} \mathrm{DM}$ & 52.2 & 50.0 & 1.10 & 0.1216 \\
\hline Gas $9 \mathrm{~h}, \mathrm{~mL} / \mathrm{g} D M$ & 87.3 & 82.7 & 1.91 & 0.1121 \\
\hline Gas $12 \mathrm{~h}, \mathrm{~mL} / \mathrm{g} \mathrm{DM}$ & 106.1 & 103.0 & 2.21 & 0.2533 \\
\hline IVDMD, \% & 40.06 & 38.73 & 1.1631 & 0.3597 \\
\hline $\mathrm{NH}_{3}-\mathrm{N}, \mathrm{mg} / 100 \mathrm{~mL}$ & 7.87 & 9.21 & 0.553 & 0.1485 \\
\hline $\mathrm{pH}$ & 5.57 & 5.69 & 0.033 & 0.0335 \\
\hline Total VFA, mM & 83.12 & 89.11 & 4.388 & 0.2592 \\
\hline Acetate, $\mathrm{mmol} / \mathrm{mol}$ & 427.50 & 448.30 & 6.359 & 0.0362 \\
\hline Propionate, $\mathrm{mmol} / \mathrm{mol}$ & 341.50 & 325.20 & 4.422 & 0.0379 \\
\hline Butyrate, $\mathrm{mmol} / \mathrm{mol}$ & 199.90 & 195.30 & 3.017 & 0.2435 \\
\hline Iso-butyrate, $\mathrm{mmol} / \mathrm{mol}$ & 1.96 & 2.16 & 0.171 & 0.3502 \\
\hline Valerate, $\mathrm{mmol} / \mathrm{mol}$ & 22.21 & 20.94 & 0.802 & 0.2752 \\
\hline Iso-valerate, $\mathrm{mmol} / \mathrm{mol}$ & 6.96 & 8.12 & 1.726 & 0.6329 \\
\hline A:P ratio & 1.25 & 1.38 & 0.033 & 0.0291 \\
\hline Total lactate, mM & 16.76 & 18.89 & 0.872 & 0.0824 \\
\hline D-lactate, mM & 9.74 & 11.26 & 0.404 & 0.0340 \\
\hline L-lactate, mM & 7.02 & 7.63 & 0.642 & 0.4552 \\
\hline
\end{tabular}

* SEM, standard error of the mean; DM, dry matter; IVDMD, in vitro dry matter digestibility; $\mathrm{NH}_{3}-\mathrm{N}$, ammonia-nitrogen; VFA, volatile fatty acids; A:P ratio, acetate to propionate ratio.

1) $\mathrm{CON}$, corn grain with elution buffer of the same volume of endolysin treatment; Ly JH307, corn grain with endolysin LyJH307 ( $0.2 \%$ of dietary DM).

The absolute abundance of total bacteria and ciliate protozoa did not show any significant differences between treatments (Table 3, total bacteria, $P=0.6036$; ciliate protozoa, $P=0.8271$ ), whereas the 
absolute abundance of $S$. bovis was significantly decreased by endolysin Ly JH307 supplementation $(P<$ 0.0289).

Table 3. Microbial absolute abundances by endolysin LyJH307 supplementation in an in vitro experiment at $12 \mathrm{~h}$ of incubation

\begin{tabular}{|c|c|c|c|c|}
\hline \multicolumn{5}{|c|}{ Treatments ${ }^{1)}$} \\
\hline Items ${ }^{*}$ & CON & LyJH307 & SEM & $P$-value \\
\hline Total bacteria ${ }^{2)}$ & 3.82 & 3.68 & 0.231 & 0.6036 \\
\hline Ciliate protozoa2) & 1.37 & 1.43 & 0.2063 & 0.8271 \\
\hline Streptococcus bovis $\left.{ }^{3}\right)$ & $5.70^{\mathrm{a}}$ & $1.76^{b}$ & 1.161 & 0.0289 \\
\hline
\end{tabular}

* SEM, standard error of the mean

1) CON, corn grain with elution buffer of the same volume of endolysin treatment; LyJH307, corn grain with endolysin Ly JH307 ( $0.2 \%$ of dietary DM).

2) $\times 10^{10}$ copies $/ \mathrm{mL}$ of rumen fluid.

3) $\times 10^{8}$ copies $/ \mathrm{mL}$ of rumen fluid.

\subsection{Effect of endolysin LyJH307 on the rumen microbiota}

In the present study, 260,866 sequences were obtained by the 16S rRNA sequence analysis with an average of $43,477 \pm 12,838$ sequences per sample. Through quality filtering by QIIME2 (Q score $>20$ ), 148,786 sequences ( $57 \%$ of the raw reads) were generated with an average of $24,797 \pm 9,238$ sequences per sample. There were no significant differences in the alpha diversity measurements, including Shannon's index, Simpson's index, Chao1 estimates, and evenness (Figure 1, Shannon's index, $P=0.5127$; Simpson's index, $P=0.5127$; Chao1 estimates, $P=0.2752$; and evenness, $P=0.1266)$ ). For the beta diversity, we presented two PCoA results based on Bray-Curtis dissimilarity and weighted UniFrac distance, followed by PERMANOVA analysis (Figure 2). The PCoA result based on Bray-Curtis dissimilarity showed statistical tendency between the CON and LyJH307 groups (Figure 2A, $P=0.0970$ ), whereas there was no significant change in the PCoA result based on the weighted UniFrac distances (Figure 2B, $P=$ 0.5030).

In the Venn diagram made by the major microbiome data, 16 of 17 phyla were shared, and 57 of 63 families were shared between the CON and LyJH307 groups. At the phylum and family levels, specific taxa were observed only in the LyJH307 group (Figure 3A and 3B). In addition, 114 of 129 genera were shared, and specific taxa in the LyJH307 group were higher than those in the CON group (Figure 3C). 
The predominant taxa with relative abundance above $0.5 \%$ in at least one group are shown in Figure 4 . At the phylum level, eight phyla had a relative abundance $>0.5 \%$, and Bacteroidota (CON, $60.9 \%$ vs. LyJH307, 56.7\%), Firmicutes (CON, 27.2\% vs. LyJH307, 27.2\%), and Proteobacteria (CON, 7.3\% vs. LyJH307, 11.2\%) were the three dominant phyla (Figure 4A), while the other phyla had a relatively minor proportion (both treatments, relative abundance $<1 \%$ ). A total of 19 families were the dominant taxa that had a relative abundance of $>0.5 \%$ (Figure $4 \mathrm{~B}$ ), and there were no significant differences in major families between the CON and LyJH307 groups. At the genus level, the three dominant genera in the CON group were Prevotellaceae (41.1\%), Rikenellaceae (11.1\%), and Lachnospiraceae (8.3\%), whereas the three dominant genera in the LyJH307 group were Prevotellaceae (39.8\%), Succinivibrionaceae (10.7\%), and Rikenellaceae (9.3\%) (Figure 4C).

The differentially abundant taxa at the phylum, family, and genus levels between the CON and LyJH307 groups were identified using LEfSe (Figure 5). At the phylum level, the relative abundance of Elusimicrobiota and Verrucomicrobiota were significantly higher in the LyJH307 group than in the CON group (Figure 5). At the family level, LyJH307 supplementation significantly increased the relative abundance of three genera within Verrucomicrobiota (WCHB1-41, vadinBE97, and Victivallaceae), one genus within Firmicutes (Lactobacillaceae), and Desulfuromonadaceae (Figure 5B). At the genus level, a total of 11 genera including Lachnoclostridium, WCHB1-41, unclassified genus Selenomonadaceae (UG_Selenomonadaceae), Paraprevotella, vadinBE97, Ruminococcusgauvreauii group, Lactobacillus, Anaerorhabdusfurcosa group, Victivallaceae, Desulfuromonadaceae, and Sediminispirochaeta had significantly higher relative abundance in the LyJH307 group than in the CON group; thus, there were no genera enriched in the CON group (Figure 5B). Among the differentially abundant genera, several genera were only detected in the LyJH307 group, including Ruminococcusgauvreauii group, Anaerorhabdusfurcosa group, Victivallaceae, Desulfuromonadaceae, and Sediminispirochaeta.

\subsection{Effect of endolysin LyJH3O7 on the predicted functions of the microbiota}

To identify the functional changes of ruminal bacteria metabolism by LyJH307 supplementation, the functional composition profiles were predicted from 16S rRNA gene sequencing data with PICRUSt2, and the various functional features were predicted from the 16S rRNA gene using five different reference databases (KEGG, Pfam, COG, enzyme classification, and MetaCyc) (Table 4). LyJH307 affected the overall increase in the numbers of total functional features (KEGG orthologs, $P=0.0559$; KEGG modules, $P=0.0535$; Pfam, $P=0.0302$; COG, $P=0.0566$; and MetaCyc pathways $P=0.0969$ ) at least showing statistical tendency (Table 4). However, the overall predicted functional features in the KEGG pathways were enriched in the CON group, with 14 KEGG pathways (other glycan degradation, ko00511; alanine, aspartate, and glutamate metabolism, ko00250; one carbon pool by folate, ko00670; amino sugar and nucleotide sugar metabolism, ko00520; zeatin biosynthesis, ko00908; biosynthesis of siderophore group nonribosomal peptides, ko01053; galactose metabolism, ko00052; glycosaminoglycan degradation, ko00531; protein digestion and absorption, ko04974; streptomycin biosynthesis, ko00521; fructose and mannose metabolism, ko00051; starch and sucrose metabolism, ko00500; pyrimidine metabolism, ko00240; and inositol phosphate metabolism, ko00562), except for biotin metabolism (ko00780) that 
was higher in the LyJH307 group (Figure 6A). In the KEGG modules, the predicted functional features were found to be enriched differently between the CON and LyJH307 groups (Figure 6B). In the CON group, keratan sulfate degradation (M00079), lipopolysaccharide transport system (M00250), ATP synthase (M00164), glycolysis (M00001), F-type ATPase (M00157), ascorbate biosynthesis (M00114), and aminoacyl-tRNA biosynthesis (M00359) were higher in the KEGG modules, whereas assimilatory sulfur reduction (M00176), PTS system (M00276), fatty acid biosynthesis (M00082), iron ( $($ ) transport system (M00190), proline biosynthesis (M00015), biotin biosynthesis (M00123), and ADP-L-glycero-Dmanno-heptose biosynthesis (M00064) were higher in the LyJH307 group (Figure 6B). Despite the changes in the low hierarchical level (KEGG pathways and modules), only two features, the biosynthesis of other secondary metabolites $(P=0.0809)$ and nucleotide metabolism $(P=0.0809)$, decreased by endolysin LyJH307 supplementation among the top 20 KEGG level 2 (Supplemental Table 1).

Table 4. Number of predicted functional features including KEGG hierarchies (orthologs, modules, and pathways), enzyme classification, MetaCyc pathways, Pfam, and clusters of orthologous genes by LyJH307 supplementation in an in vitro experiment at $12 \mathrm{~h}$ of incubation

\begin{tabular}{|lllll|}
\hline \multicolumn{5}{|c|}{ Treatments $^{1)}$} \\
Items \\
${ }^{*}$ & CON & LyJH307 & SEM & $P$-value \\
\hline KEGG orthologs & 5137 & 5354 & 60.281 & 0.0559 \\
\hline KEGG modules & 270 & 277 & 2.082 & 0.0535 \\
\hline KEGG pathways & 137 & 142 & 3.215 & 0.2381 \\
\hline Pfam & 5954 & 6254 & 75.508 & 0.0302 \\
\hline COG & 3887 & 4007 & 36.226 & 0.0566 \\
\hline EC & 1603 & 1656 & 21.759 & 0.137 \\
\hline MetaCyc pathways & 328 & 339 & 3.756 & 0.0969 \\
\hline
\end{tabular}

* SEM, standard error of the mean; KEGG, Kyoto Encyclopedia of Genes and Genomes; COG, clusters of orthologous genes; EC, enzyme classification.

1) $\mathrm{CON}$, corn grain with elution buffer of the same volume of endolysin treatment; LyJH307, corn grain with recombinant LyJH307 ( $0.2 \%$ of dietary DM).

\subsection{Correlations between microbial taxa and ruminal fermentation characteristics}

Strong correlations $(|| \mid>0.8, P<0.05)$ between ruminal fermentation characteristics and differently abundant taxa analyzed by LEfSe were detected (Figure 7). Lactobacillaceae, Desulfuromonadaceae (family), Ruminococcusgauvreauii group, Lactobacillus, and Desulfuromonadaceae (genus), all of which were higher in the $\mathrm{Ly} \mathrm{JH} 307$ group than in the CON group, were positively correlated with ruminal $\mathrm{pH}$. In 
the individual VFA proportions, Elusimicrobiota, Desulfuromonadaceae (family), Lachnoclostridium, unclassified genus (UG)_Selenomonadaceae, Ruminococcusgauvreauii group, and Desulfuromonadaceae (genus) were all increased in the Ly JH307 group and were positively correlated with the acetate proportion and negatively correlated with that of propionate. Verrucomicrobiota, WCHB141, Lactobacillaceae, Lachnoclostridium, WCHB1-41, UG_Selenomonadaceae, Paraprevotella, and Lactobacillus were positively correlated with D-lactate concentration.

\section{Discussion}

The rumen is a distinct digestive organ that degrades plant originated nutrients that are non-edible to mono-gastric animals and symbiotic ecosystem-living anaerobic bacteria, archaea, protozoa, and fungi sharing fermentation metabolites. Therefore, artificial modulation of rumen fermentation is challenging. Previously, several studies observed that supplementation with ionophore antibiotics could decrease the ruminal bacteria (especially gram-positive bacteria) in a high concentrate diet, thereby preventing a severe decrease of ruminal $\mathrm{pH}$ [30-32]. However, the potential for antibiotic resistance in ruminal bacteria has made it impossible to use ionophore antibiotics as a feed additive to modulate rumen fermentation [33, 34]. The present study is the first trial representing a new strategy to control $S$. bovis specifically using endolysin. This study confirmed that endolysin LyJH307 supplementation decreased the absolute abundance of $S$. bovis efficiently, thereby increasing ruminal $\mathrm{pH}$ in an in vitro batch culture.

Previously, we observed that the same dosage of endolysin LyJH307 used in the present study did not significantly change the absolute abundance of $S$. bovis and rumen fermentation parameters (including $\mathrm{pH}$, IVDMD, and VFA) when the endolysin LyJH307 was added at the start of the incubation in an in vitro system (data not shown). Therefore, we hypothesized that the supplementation time is important to induce adequate lytic activity in an in vitro system. In the present study, endolysin LyJH307 supplementation at $6 \mathrm{~h}$ incubation time markedly decreased the absolute abundance of $S$. bovis (approximately $70 \%$ ), and the reduction rate of $S$. bovis in the present study was similar to previous results presenting reduction rates of the $S$. bovis group ( $S$. equinus, $S$. gallolyticus subspecies pasteurianus, $S$. alactolyticus, and $S$. infantarius subspecies infantarius) in single cultures [11]. Therefore, the present dosage of endolysin LyJH307 was appropriate for the control of S. bovis under ruminal mixed culture conditions. However, there was a discrepancy between the two experiments related to dosing time ( 0 vs. 6 h). Endolysin LyJH307 is a small protein ( $32 \mathrm{kDa})$ and has high solubility among a wide range of $\mathrm{pH}(5.0-6.5)$, temperature $\left(25-55^{\circ} \mathrm{C}\right)$, and $\mathrm{NaCl}$ concentration $(0-500 \mathrm{mM})$. In the rumen, protein sources can be degraded by various bacteria such as P. ruminicola, S. ruminantium, Butyrivibrio fibrisolvens, Clostridium proteoclasticus, and Eubacterium ruminantium [35], and the solubility of proteins is one of the key factors for determining the degradability of protein [36]. Therefore, it was assumed that the application of endolysin to control specific ruminal bacteria was a possible strategy; however, the administration timing must be explored because of the potential of endolysin degradation.

Considering that LyJH307 can kill S. bovis that ferments glucose to lactate rapidly under high starch diet conditions, it was expected that LyJH307 supplementation would maintain the overall fermentation 
parameters (gas production, IVDMD, and VFA production) with increasing ruminal $\mathrm{pH}$ when only corn was used as an experimental diet. LyJH307 significantly increased the ruminal pH without any negative effects on the overall fermentation parameters. One of the standard points of ruminal pH for SARA is below 5.6 and that of ruminal pH for acute acidosis is below 5.0 [1]. In the present study, although the pH of both treatments was close to SARA conditions rather than acute acidosis (CON group, 5.57 vs. LyJH307 group, 5.69), it was assumed that a decrease in the $S$. bovis might be related to an increased ruminal $\mathrm{pH}$ in the LyJH307 supplemented group. Several agents to inhibit ruminal bacteria were evaluated in an in vitro system, and the ability to change the rumen microbiota and rumen fermentation has been shown [37-39]. Shen, Liu, Yu (39) reported that monensin and nisin supplementation decreased the absolute abundance of total bacteria and overall rumen fermentation (gas and VFA production) with a concomitant change in the major rumen microbiota. The supplementation of plant secondary metabolites such as thymol and oregano oil has recently shown to change the major microbiota with a concomitant reduction in feed digestion and rumen fermentation [38, 40]. In the present study, endolysin LyJH307 did not affect the absolute abundance of total bacteria, ciliate protozoa (Table 3 ) and major microbiota from phylum to genus levels (having relative proportions $>1 \%$ ) (Figure 4). Prevotella was the most abundant genus of Bacteroidota and Succiniclasticum was that of Firmicutes in the present study. Prevotella is the most predominant ruminal genus [41], and many are related to the digestion of polysaccharides and protein [42]. Succiniclasticum is known for its role in converting succinate to propionate [43], and previous studies have shown that the relative proportion of Succiniclasticum can be increased in a high grain diet, in agreeance with our findings $[44,45]$. The relative proportion of Verrucomicrobiota was higher in animals fed a low grain diet than those fed a high grain diet [46, 47], and rumen under normal pH contained a higher Verrucomicrobiota proportion than those undergoing SARA [48]. In the present study, the relative proportion of Verrucomicrobiota was higher in the LyJH307 group, which showed higher ruminal $\mathrm{pH}$ compared to that of the $\mathrm{CON}$. Therefore, we postulated that Verrucomicrobiota might be used as one of the potential biomarkers indicating the SARA condition. In the minor taxa at the genus level, 11 genera with a relative proportion $<0.1 \%$ were increased by endolysin $\mathrm{LyJH307}$, including Lachnoclostridium, WCHB1-41, UG_Selenomonadaceae, Paraprevotella, vadinBE97, Ruminococcusgauvreauii group, Lactobacillus, Anaerohabdusfurcosa group, Victivallaceae, Desulfuromonadaceae, and Sediminispirochaeta. In a previous study, endolysin Ly JH307 had a narrow range of lytic activity, specifically in the S. bovis group [11]. The narrow specificity of LyJH307 to kill target bacteria might lead to an increase of minor microbial taxa competing with $S$. bovis to use starch carbohydrates; therefore, no significant changes in rumen fermentation (gas, VFA, and IVDMD) were observed.

In the rumen, VFA is an end-product produced by microbial fermentation, and the proportions of individual VFA are related to dietary characteristics by microbes [49]. Therefore, changes in individual VFA proportions mean changes in the microbiota. In the present study, endolysin LyJH307 induced an increase in acetate proportion but a decrease in propionate proportion, thus increasing the A:P ratio. The increased proportion of acetate in the $\mathrm{LyJH} 307$ group can be explained by the increase of several minor bacterial genera, such as Lachnoclostridium, Paraprevotella, Ruminococcusgauvreauii group, and 
Sediminispirochaeta. The Lachnoclostridium and Ruminococcusgauvreauii group are gram-positive and obligate anaerobic bacteria that are known for producing acetate as the main end-product of glucose fermentation [50,51]. Previously, Paraprevotella and Sediminispirochaeta were reported to produce acetate as the end product of glucose metabolism $[52,53]$. Thus, the reduction of $S$. bovis by supplementation of endolysin LyJH307 might induce the increase of several minor genera producing acetate as the main fermentation product. Lachnoclostridium, UG_Selenomonadaceae, Ruminococcusgauvreauii_group, and Desulfuromonadaceae (genus) that were higher in the LyJH307 group than in the CON group were positively correlated with acetate proportion and negatively correlated with propionate proportion. Little is known about the function of UG_Selenomonadaceae and Desulfuromonadaceae (genus) in producing acetate in the rumen, therefore further research is required to determine their relationship with acetate and propionate proportion.

S. bovis produces lactate as the major fermentation product when the starch source is enriched in the diet [54], and this bacterial species can generate more ATP per hour than the other ruminal amylolytic bacteria [55]. In the present study, endolysin LyJH307 supplementation increased D-lactate concentration with a concomitant increase in total lactate production. Lactobacillus is generally connected with acute acidosis and SARA because of its ability to produce lactate as the main fermentation product and high acid resistance [1]. Lactobacillus can digest starch sources including corn and barley under non-acidosis conditions [56]. Therefore, the increased proportion of Lactobacillus in the LyJH307 group might be related to the increased availability of a starch source achieved by decreasing the abundance of $S$. bovis. Previous studies have shown that several species of Selenomonas can produce lactate as the main fermentation product with high concentrations of glucose, whereas the major fermentation products are changed to acetate and propionate in a glucose-limited condition [57-59]. In the present study, considering that the feed ingredient was only corn grain, it was speculated that the diet might affect the alteration in the major fermentation product of UG_Selenomonadaceae to lactate, and the increase of UG_Selenomonadaceae in LyJH307 group might cause an increase in the D-lactate concentration. In addition, Lachnoclostridium, WCHB1-41, UG_Selenomonadaceae, Paraprevotella, and Lactobacillus, which were increased in the LyJH307 group, were positively correlated with D-lactate concentration, therefore they might have also contributed to the high D-lactate concentration in the LyJH307 group. However, since correlation does not mean causation between factors, further research is required to determine their relationship with D-lactate production.

The alteration of ruminal microbiota composition by using dietary feed additives is linked with a change of microbial functional features. In the present study, PICRUSt2 was used to predict the functional changes of ruminal microbiota associated with LyJH307 supplementation. The addition of endolysin LyJH307 altered several bacterial populations that had a minor relative proportion $(<1 \%)$ across all samples, in addition to the similar alpha-diversity measurements across the treatments, the number of predicted functional features (KEGG orthologs, KEGG modules, PFAM, COG, and MetaCyc pathways) was tended to be higher in endolysin treatment. However, among the differentially abundant KEGG pathways between the CON and LyJH307 groups analyzed by LEfSe, the KEGG pathways involved in carbohydrate metabolism (e.g., starch and sucrose metabolism, amino sugar and nucleotide sugar metabolism, 
fructose and mannose metabolism, and galactose metabolism, and inositol phosphate metabolism) were abundant in the CON group. Thus, the addition of endolysin Ly JH307 might change carbohydrate metabolism with concomitant changes in minor rumen microbiota, thereby inducing higher ruminal pH in the LyJH307 group than in the CON group.

Previously, Bhatt, Mohapatra, Anand, Kuntal and Mande (60) reported the possibility of incorrect assignments in KEGG modules related to bacterial systems, leading to misinterpretation. Ascorbate biosynthesis plants, (glucose-6-phosphate to ascorbate) (M00114), which was higher in the CON group than in the LyJH307 group, is a plant metabolism pathway related to the formation of L-ascorbate from GDP-D-mannose. KEGG orthologs contained ascorbate biosynthesis, plants, (glucose-6P to ascorbate) in the present study entry were mannose-1-phosphate guanylyltransferase and mannose-6-phosphate isomerase, which are enzymes converting GDP-D-mannose to fructose-6-phosphate. Thus, the module definition of ascorbate biosynthesis, plants, (glucose-6-phosphate to ascorbate) in the present study was misused as previously suggested [60].

F-type ATPase is the most common ATPase/synthase found in bacteria, and it generally acts as ATP synthase driving ATP synthesis through a passive flux of $\mathrm{H}^{+}$in aerobic organisms [61]. Several studies have shown that F-type ATPase can be used for ATP synthase by translocation of $\mathrm{H}^{+}$and $\mathrm{Na}^{+}$in strict anaerobes [62-64]. Lu, Xu, Shen, Tian and Shen (65) showed that the F-type ATPase was significantly increased with an increase in dietary grain level in the diet. In the present study, considering that glycolysis (M00001), ATP synthase (M00164), and F-type ATPase (M00157) were higher in the CON group than in the LyJH307 group, the use of endolysin LyJH307 might induce reduction of efficiency in carbohydrate availability by specifically inhibition of $S$. bovis.

\section{Conclusion}

In summary, endolysin LyJH307 supplementation markedly decreased the absolute abundance of $S$. bovis and improved the ruminal $\mathrm{pH}$ thereby eliminating any negative effects on overall fermentation parameters (gas, IVDMD, and total VFA) in an in vitro experiment. In the $16 \mathrm{~S}$ amplicon sequencing analysis, endolysin LyJH307 caused a shift in minor rumen microbiota and its metabolic pathways related to carbohydrate metabolism, and these changes were associated with the increase of acetate proportion and D-lactate concentration. This study provides the first insight into the availability of endolysin as a specific modulator for rumen and shows the possibility of endolysin degradation by rumen microbiota. These findings provide new perspectives for modulating ruminal digestion and disease causative bacteria by using endolysin in the rumen.

\section{Declarations}

\section{Authors' contributions}


$\mathrm{JS}, \mathrm{HK}$, and IK conceived the experiment. HK conducted the in vitro experiment and analyzed the microbial sequencing data. HK, TP, and JS wrote the manuscript. HK, TP, IK, and JS reviewed the manuscript and read and approved the final manuscript.

\section{Funding}

This work was supported by the National Research Foundation of Korea (NRF) grant funded by the Korea government (MSIT) (No. 2019R1F1A1056904). The funders did not play any role in this study or in the preparation of the manuscript.

\section{Availability of data and materials}

The raw 16S rRNA sequences have been deposited in the NCBI SRA under BioProject PRJNA699718. All analyzed microbial datasets in the present study are available from the corresponding author on reasonable request.

\section{Ethics approval and consent to participate}

Not applicable.

\section{Consent for publication}

All the authors read and agree to the content of this paper and its publication.

\section{Competing interests}

The authors declare no competing interest.

\section{Acknowledgements}

None.

\section{Abbreviations}

aNDF: neutral detergent fiber

ASVs: amplicon sequencing variants

A:P ratio: acetate to propionate ratio

CBD: C-terminal cell wall binding domain

CON: control diet

CP: crude protein 
DM: dry matter

EAD: N-terminal enzymatically active domain

IVDMD: in vitro dry matter digestibility

KEGG: Kyoto Encyclopedia of Genes and Genomes

LEfSe: linear discriminant analysis effect size

LyJH307: endolysin LyJH307 treatment

$\mathrm{NH}_{3}-\mathrm{N}$ : ammonia-nitrogen

NFC: non-fiber carbohydrate

PERMANOVA: permutation multivariate analysis of variance

PG: peptidoglycan

PCoA: principal coordinate analysis

QIIME2: Quantitative Insights into Microbial Ecology 2

SARA: subacute ruminal acidosis

VFA: volatile fatty acids

\section{References}

1. Nagaraja T, Lechtenberg KF. Acidosis in feedlot cattle. Veterinary Clinics of North America: Food Animal Practice. 2007;23:333-50.

2. Nagaraja TG, Titgemeyer EC. Ruminal acidosis in beef cattle: The current microbiological and nutritional outlook1, 2. Journal of Dairy Science. 2007;90:E17-E38.

3. Kung Jr L, Hession AO. Preventing in vitro lactate accumulation in ruminal fermentations by inoculation with megasphaera elsdenii. Journal of Animal Science. 1995;73:250-6.

4. Russell J, Robinson P. Compositions and characteristics of strains of streptococcus bovis. Journal of Dairy Science. 1984;67:1525-31.

5. Chow J, Russell J. Effect of ionophores and ph on growth of streptococcus bovis in batch and continuous culture. Applied and environmental microbiology. 1990;56:1588-93.

6. Bevans D, Beauchemin K, Schwartzkopf-Genswein K, McKinnon J, McAllister T. Effect of rapid or gradual grain adaptation on subacute acidosis and feed intake by feedlot cattle. Journal of Animal Science. 2005;83:1116-32. 
7. Krause K, Dhuyvetter D, Oetzel G. Effect of a low-moisture buffer block on ruminal ph in lactating dairy cattle induced with subacute ruminal acidosis. Journal of dairy science. 2009;92:352-64.

8. Blanch M, Calsamiglia S, Devant M, Bach A. Effects of acarbose on ruminal fermentation, blood metabolites and microbial profile involved in ruminal acidosis in lactating cows fed a highcarbohydrate ration. The Journal of Dairy Research. 2010;77:123.

9. Schmelcher M, Donovan DM, Loessner MJ. Bacteriophage endolysins as novel antimicrobials. Future microbiology. 2012;7:1147-71.

10. Loessner MJ. Bacteriophage endolysins-current state of research and applications. Current opinion in microbiology. 2005;8:480-7.

11. Kim H, Lee HG, Kwon I, Seo J. Characterization of endolysin lyjh307 with antimicrobial activity against streptococcus bovis. Animals. 2020;10:963.

12. Obeso JM, Martínez B, Rodríguez A, García P. Lytic activity of the recombinant staphylococcal bacteriophage $\varphi$ h5 endolysin active against staphylococcus aureus in milk. International journal of food microbiology. 2008;128:212-8.

13. Swift SM, Seal BS, Garrish JK, Oakley BB, Hiett K, Yeh H-Y, et al. A thermophilic phage endolysin fusion to a clostridium perfringens-specific cell wall binding domain creates an anti-clostridium antimicrobial with improved thermostability. Viruses. 2015;7:3019-34.

14. Love MJ, Bhandari D, Dobson RC, Billington C. Potential for bacteriophage endolysins to supplement or replace antibiotics in food production and clinical care. Antibiotics. 2018;7:17.

15. Fan J, Zeng Z, Mai K, Yang Y, Feng J, Bai Y, et al. Preliminary treatment of bovine mastitis caused by staphylococcus aureus, with trx-sa1, recombinant endolysin of s. Aureus bacteriophage ime-sa1. Veterinary microbiology. 2016;191:65-71.

16. Van Tassell ML, Ibarra-Sánchez LA, Hoepker GP, Miller MJ. Hot topic: Antilisterial activity by endolysin plyp100 in fresh cheese. Journal of dairy science. 2017;100:2482-7.

17. AOAC. Official methods of analysis of the association of analytical chemists international: Association of Official Analytical Chemists International; 2000.

18. AOAC. Official methods of analysis of the association of analytical chemists international. Official methods of analysis of AOAC International, Gaithersburg: Maryland. 2006;200.

19. Van Soest Pv, Robertson J, Lewis B. Methods for dietary fiber, neutral detergent fiber, and nonstarch polysaccharides in relation to animal nutrition. Journal of dairy science. 1991;74:3583-97.

20. Goering HK, Van Soest PJ. Forage fiber analyses (apparatus, reagents, prcedures, and some applications). USDA Agr Handb. 1970.

21. Theodorou MK, Williams BA, Dhanoa MS, McAllan AB, France J. A simple gas production method using a pressure transducer to determine the fermentation kinetics of ruminant feeds. Animal feed science and technology. 1994;48:185-97.

22. Chaney AL, Marbach EP. Modified reagents for determination of urea and ammonia. Clinical chemistry. 1962;8:130-2. 
23. Yu Z, Morrison M. Improved extraction of pcr-quality community DNA from digesta and fecal samples. Biotechniques. 2004;36:808-12.

24. Kim H, Kim B, Cho S, Kwon I, Seo J. Dietary lysophospholipids supplementation inhibited the activity of lipolytic bacteria in forage with high oil diet: An in vitro study. Asian-Australasian Journal of Animal Sciences. 2020.

25. Bolyen E, Rideout JR, Dillon MR, Bokulich NA, Abnet C, Al-Ghalith GA, et al. Qiime 2: Reproducible, interactive, scalable, and extensible microbiome data science. PeerJ Preprints, 2018 2167-9843.

26. Gordon A, Hannon G. Fastx toolkit. 2010.

27. Magoč T, Salzberg SL. Flash: Fast length adjustment of short reads to improve genome assemblies. Bioinformatics. 2011;27:2957-63.

28. Quast C, Pruesse E, Yilmaz P, Gerken J, Schweer T, Yarza P, et al. The silva ribosomal rna gene database project: Improved data processing and web-based tools. Nucleic acids research. 2012;41:D590-D6.

29. Douglas GM, Maffei VJ, Zaneveld J, Yurgel SN, Brown JR, Taylor CM, et al. Picrust2: An improved and extensible approach for metagenome inference. BioRxiv. 2019:672295.

30. Dennis S, Nagaraja T, Bartley E. Effect of lasalocid or monensin on lactate-producing or using rumen bacteria. Journal of Animal Science. 1981;52:418-26.

31. Nagaraja T, Avery T, Galitzer S, Harmon D. Effect of ionophore antibiotics on experimentally induced lactic acidosis in cattle. American Journal of Veterinary Research. 1985;46:2444-52.

32. Newbold CJ, Wallace RJ, Watt N, Richardson AJ. Effect of the novel ionophore tetronasin (ici 139603) on ruminal microorganisms. Applied and Environmental Microbiology. 1988;54:544-7.

33. Park SY, Lee M, Lim SR, Kwon H, Lee YS, Kim JH, et al. Diversity and antimicrobial resistance in the streptococcus bovis/streptococcus equinus complex (sbsec) isolated from korean domestic ruminants. Microorganisms. 2021;9:98.

34. Russell JB, Houlihan AJ. Ionophore resistance of ruminal bacteria and its potential impact on human health. FEMS microbiology reviews. 2003;27:65-74.

35. Reilly K, Carruthers V, Attwood GT. Design and use of $16 \mathrm{~s}$ ribosomal DNA-directed primers in competitive pcrs to enumerate proteolytic bacteria in the rumen. Microbial Ecology. 2002;43:259-70.

36. Bach A, Calsamiglia S, Stern M. Nitrogen metabolism in the rumen. Journal of dairy science. 2005;88:E9-E21.

37. Hassan F-u, Arshad MA, Ebeid HM, Rehman MS-u, Khan MS, Shahid S, et al. Phytogenic additives can modulate rumen microbiome to mediate fermentation kinetics and methanogenesis through exploiting diet-microbe interaction. Frontiers in Veterinary Science. 2020;7.

38. Zhou R, Wu J, Lang X, Liu L, Casper DP, Wang C, et al. Effects of oregano essential oil on in vitro ruminal fermentation, methane production, and ruminal microbial community. Journal of Dairy Science. 2020;103:2303-14. 
39. Shen J, Liu Z, Yu Z, Zhu W. Monensin and nisin affect rumen fermentation and microbiota differently in vitro. Frontiers in microbiology. 2017;8:1111.

40. Yu J, Cai L, Zhang J, Yang A, Wang Y, Zhang L, et al. Effects of thymol supplementation on goat rumen fermentation and rumen microbiota in vitro. Microorganisms. 2020;8:1160.

41. Bekele AZ, Koike S, Kobayashi Y. Genetic diversity and diet specificity of ruminal prevotella revealed by 16 s rna gene-based analysis. FEMS microbiology letters. 2010;305:49-57.

42. Accetto T, Avguštin G. Polysaccharide utilization locus and cazyme genome repertoires reveal diverse ecological adaptation of prevotella species. Systematic and applied microbiology. 2015;38:453-61.

43. Van Gylswyk N. Succiniclasticum ruminis gen. Nov., sp. Nov., a ruminal bacterium converting succinate to propionate as the sole energy-yielding mechanism. International Journal of Systematic and Evolutionary Microbiology. 1995;45:297-300.

44. Liu J-h, Bian G-r, Zhu W-y, Mao S-y. High-grain feeding causes strong shifts in ruminal epithelial bacterial community and expression of toll-like receptor genes in goats. Frontiers in microbiology. 2015;6:167.

45. Petri R, Schwaiger T, Penner G, Beauchemin K, Forster R, McKinnon J, et al. Changes in the rumen epimural bacterial diversity of beef cattle as affected by diet and induced ruminal acidosis. Applied and environmental microbiology. 2013;79:3744-55.

46. Hua C, Tian J, Tian P, Cong R, Luo Y, Geng Y, et al. Feeding a high concentration diet induces unhealthy alterations in the composition and metabolism of ruminal microbiota and host response in a goat model. Frontiers in microbiology. 2017;8:138.

47. Park T, Ma L, Ma Y, Zhou X, Bu D, Yu Z. Dietary energy sources and levels shift the multi-kingdom microbiota and functions in the rumen of lactating dairy cows. Journal of Animal Science and Biotechnology. 2020;11:1-16.

48. Plaizier JC, Li S, Danscher AM, Derakshani H, Andersen PH, Khafipour E. Changes in microbiota in rumen digesta and feces due to a grain-based subacute ruminal acidosis (sara) challenge. Microbial ecology. 2017;74:485-95.

49. Dijkstra J. Production and absorption of volatile fatty acids in the rumen. Livestock Production Science. 1994;39:61-9.

50. Domingo M-C, Huletsky A, Boissinot M, Bernard KA, Picard FJ, Bergeron MG. Ruminococcus gauvreauii sp. Nov., a glycopeptide-resistant species isolated from a human faecal specimen. International Journal of Systematic and Evolutionary Microbiology. 2008;58:1393-7.

51. Yutin N, Galperin MY. A genomic update on clostridial phylogeny: G ram-negative spore formers and other misplaced clostridia. Environmental microbiology. 2013;15:2631-41.

52. Morotomi M, Nagai F, Sakon H, Tanaka R. Paraprevotella clara gen. Nov., sp. Nov. And paraprevotella xylaniphila sp. Nov., members of the family 'prevotellaceae' isolated from human faeces. International Journal of Systematic and Evolutionary Microbiology. 2009;59:1895-900.

53. Shivani Y, Subhash Y, Sasikala C, Ramana CV. Description of 'candidatus marispirochaeta associata' and reclassification of spirochaeta bajacaliforniensis, spirochaeta smaragdinae and spirochaeta 
sinaica to a new genus sediminispirochaeta gen. Nov. As sediminispirochaeta bajacaliforniensis comb. Nov., sediminispirochaeta smaragdinae comb. Nov. And sediminispirochaeta sinaica comb. Nov. International Journal of Systematic and Evolutionary Microbiology. 2016;66:5485-92.

54. Russell JB, Hino T. Regulation of lactate production in streptococcus bovis: A spiraling effect that contributes to rumen acidosis. Journal of Dairy Science. 1985;68:1712-21.

55. Hungate RE. Evolution of a microbial ecologist. Annual Reviews in Microbiology. 1979;33:1-21.

56. Yang HE, Zotti CA, McKinnon JJ, McAllister TA. Lactobacilli are prominent members of the microbiota involved in the ruminal digestion of barley and corn. Frontiers in microbiology. 2018;9:718.

57. Scheifinger C, Latham M, Wolin M. Relationship of lactate dehydrogenase specificity and growth rate to lactate metabolism by selenomonas ruminantium. Appl Microbiol. 1975;30:916-21.

58. Melville SB, Michel T, Macy JM. Pathway and sites for energy conservation in the metabolism of glucose by selenomonas ruminantium. Journal of bacteriology. 1988;170:5298-304.

59. Wallace RJ. Control of lactate production by selenomonas ruminantium: Homotropic activation of lactate dehydrogenase by pyruvate. Microbiology. 1978;107:45-52.

60. Bhatt V, Mohapatra A, Anand S, Kuntal BK, Mande SS. Flim-map: Gene context based identification of functional modules in bacterial metabolic pathways. Frontiers in microbiology. 2018;9:2183.

61. Ozawa K, Meikari T, Motohashi K, Yoshida M, Akutsu H. Evidence for the presence of an f-type atp synthase involved in sulfate respiration in desulfovibrio vulgaris. Journal of bacteriology. 2000;182:2200-6.

62. Dimroth P. Primary sodium ion translocating enzymes. Biochimica et Biophysica Acta (BBA)Bioenergetics. 1997;1318:11-51.

63. Fritz $\mathrm{M}$, Müller V. An intermediate step in the evolution of atpases- the $\mathrm{f} 1 \mathrm{f0}$-atpase from acetobacterium woodii contains f-type and v-type rotor subunits and is capable of atp synthesis. The FEBS journal. 2007;274:3421-8.

64. Das A, Ljungdahl LG. Composition and primary structure of the $\mathrm{f} 1 \mathrm{f0}$ atp synthase from the obligately anaerobic bacterium clostridium thermoaceticum. Journal of Bacteriology. 1997;179:3746-55.

65. Lu Z, Xu Z, Shen Z, Tian Y, Shen H. Dietary energy level promotes rumen microbial protein synthesis by improving the energy productivity of the ruminal microbiome. Frontiers in microbiology. 2019;10:847.

66. Denman SE, McSweeney CS. Development of a real-time pcr assay for monitoring anaerobic fungal and cellulolytic bacterial populations within the rumen. FEMS microbiology ecology. 2006;58:572-82.

67. Sylvester JT, Karnati SK, Yu Z, Morrison M, Firkins JL. Development of an assay to quantify rumen ciliate protozoal biomass in cows using real-time pcr. The Journal of Nutrition. 2004;134:3378-84.

68. Khafipour E, Li S, Plaizier JC, Krause DO. Rumen microbiome composition determined using two nutritional models of subacute ruminal acidosis. Applied and environmental microbiology. 2009;75:7115-24. 

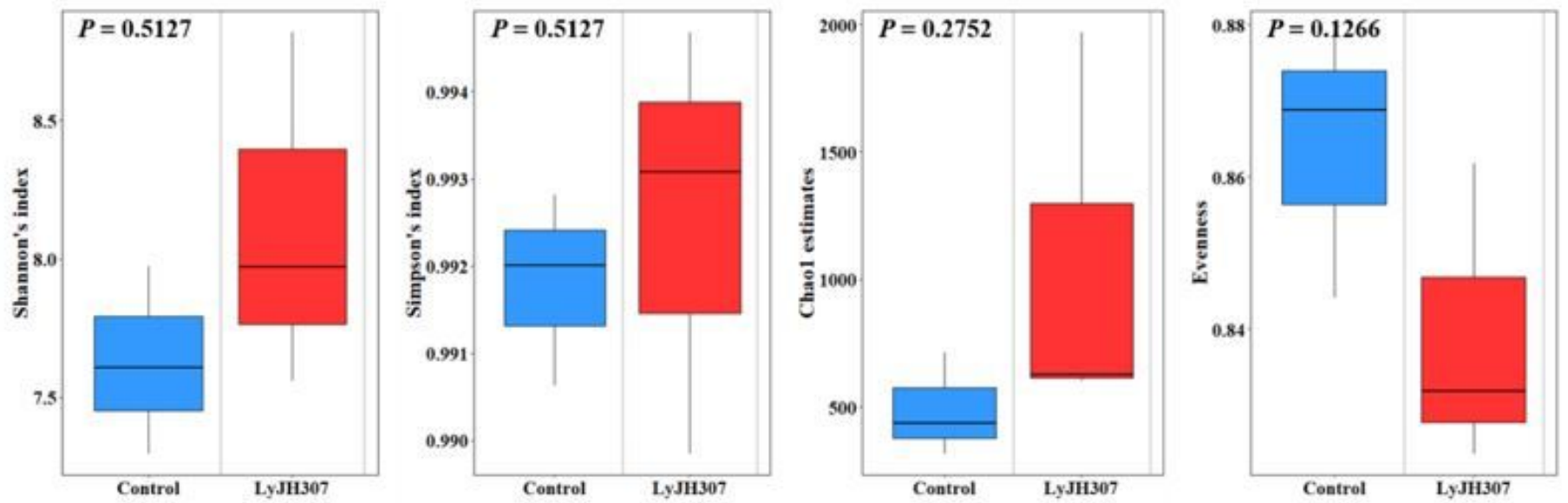

\section{Figure 1}

Alpha diversity indices after endolysin LyJH307 supplementation in an in vitro experiment at $12 \mathrm{~h}$ of incubation.

(A) Bray-Curtis dissimilarity $(P=0.097)$

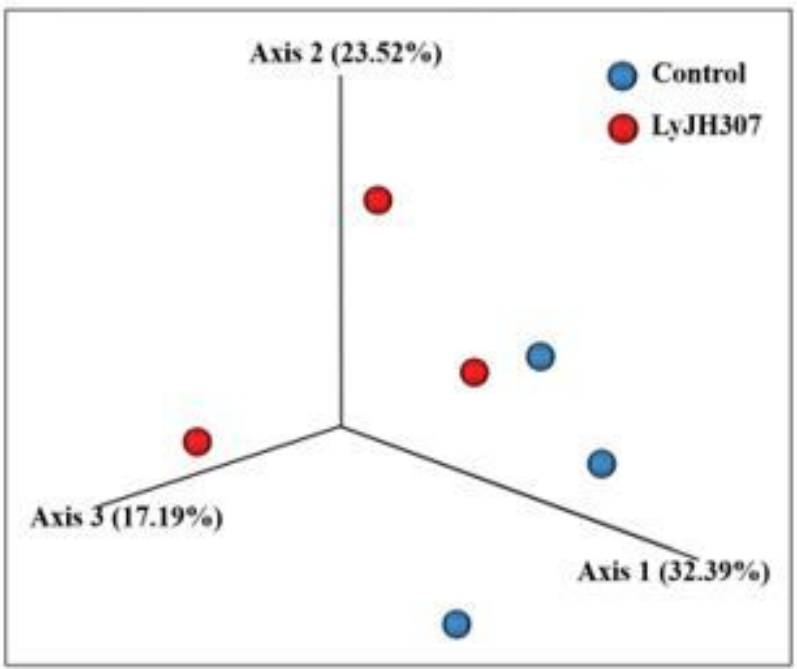

(B) Weighted UniFrac $(P=0.503)$

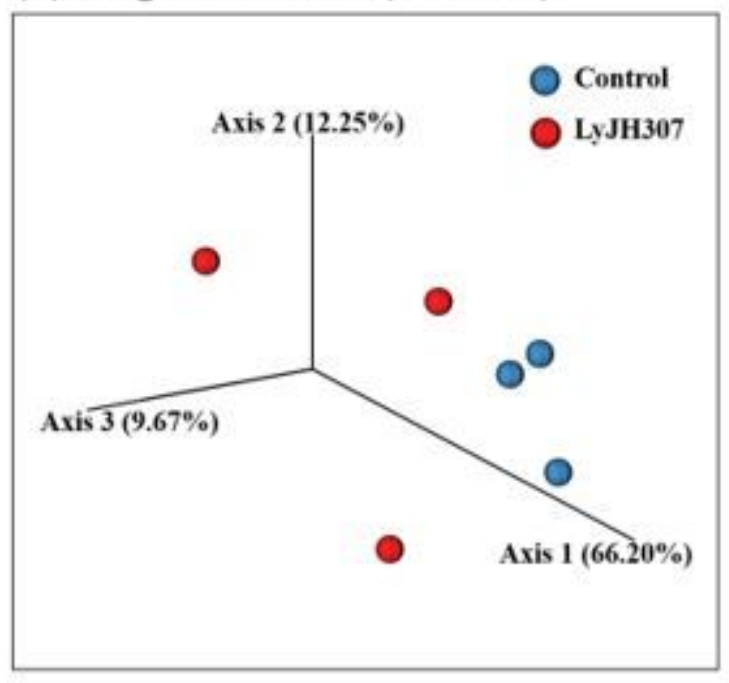

\section{Figure 2}

Principal coordinates analysis based on (A) Bray-Curtis dissimilarity and (B) weighted UniFrac distances. PERMANOVA analysis was used for comparing differences between the control and LyJH307 groups. 
(A) Phylum level

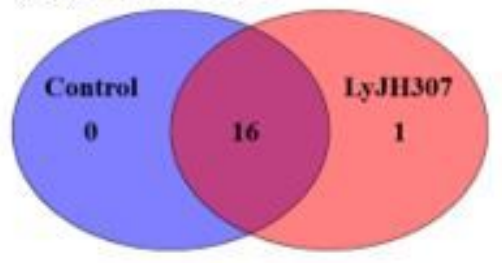

(B) Family level

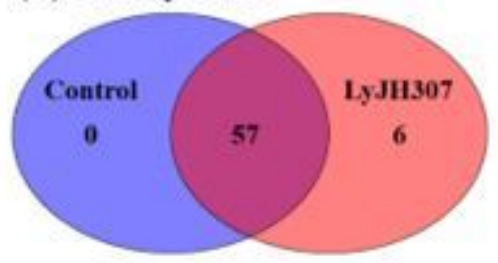

(C) Genus level

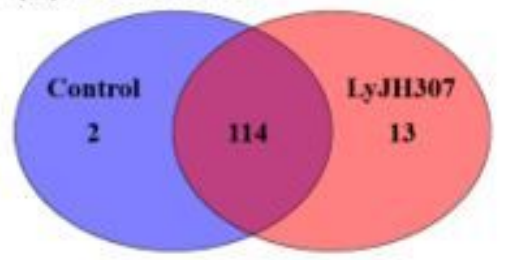

Figure 3

Venn diagram of commonly and exclusively identified prokaryotic taxa in collapsed BIOM table for (A) phylum, (B) family, and (C) genus levels.

(A) Phylum level

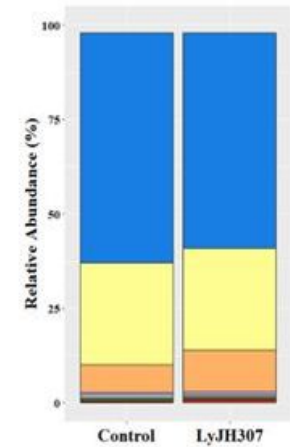

(B) Family level

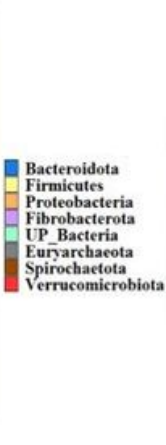

\section{(B) Family level}

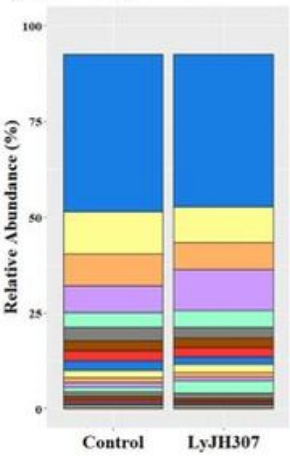

(C) Genus level

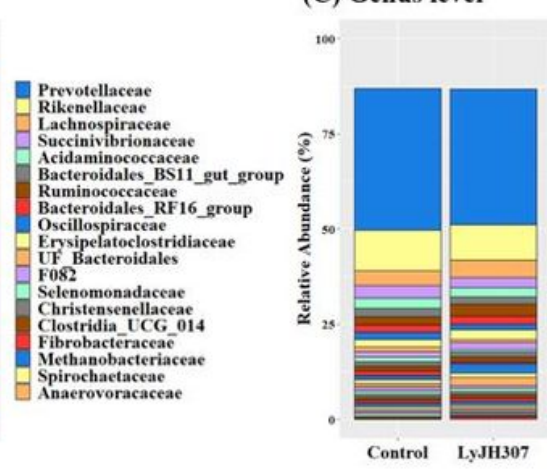

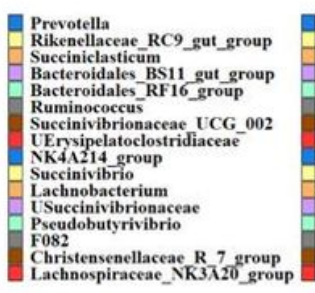

- Ruminobacter 2 Prevotellaceae_UCG_001 Selenomonas - $2 C$ _ 001 lostridia_UCG-014 Prevotellaceae_YAB2003_group Prevotellaceace Lachnospiraceae
revotellaterea_UCG_003 Fibrobacter Succinimonas
Acetitomaculum Suttleworthia achnoclostridium Iethanobrevibacter
Anaerovibrio

\section{Figure 4}

Distribution of the predominant rumen microbiota at (A) phylum, (B) family, and (C) genus levels with each proportion above $0.5 \%$ and found in at least $50 \%$ the samples in each treatment. 
(A)
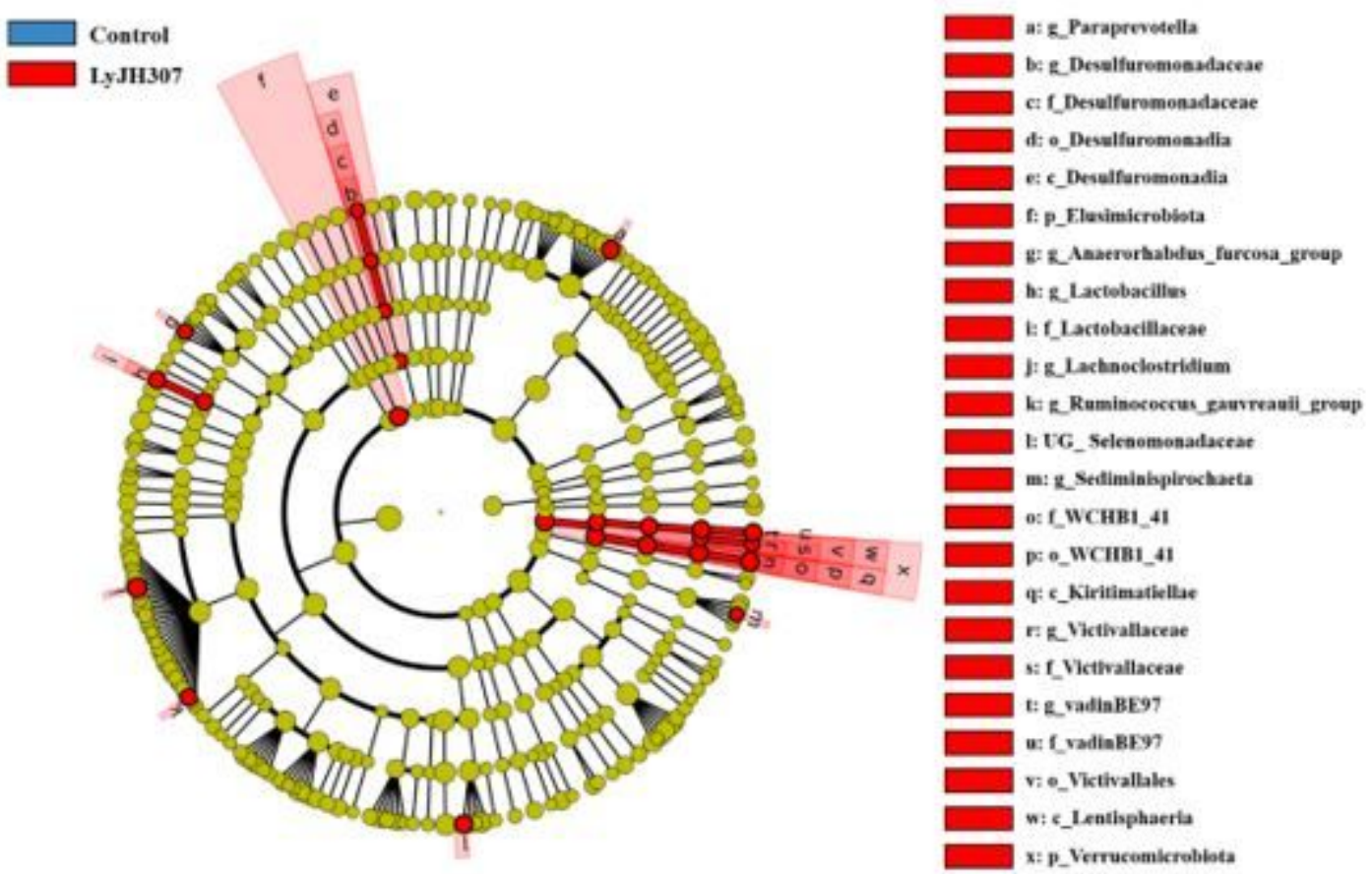

(B)

\begin{abstract}
Control
LyJH307
\end{abstract}

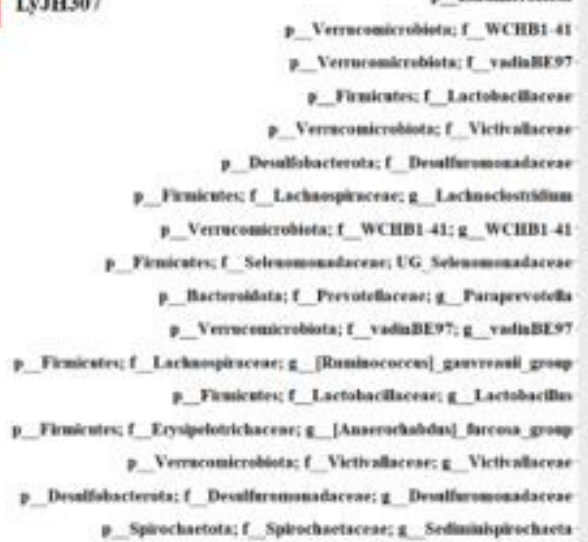

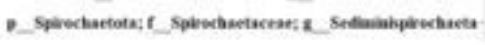

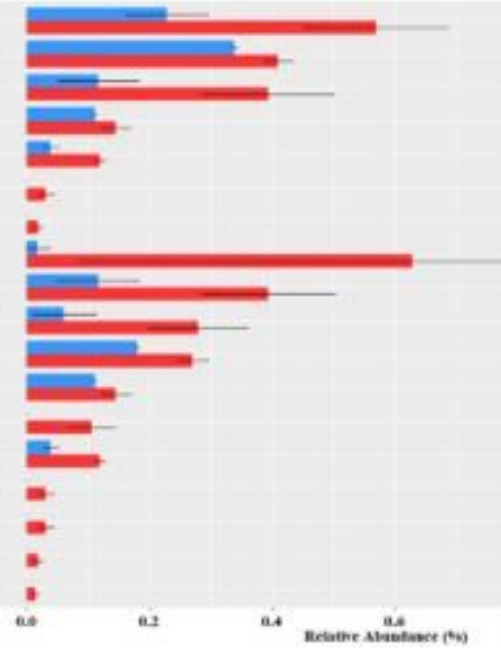

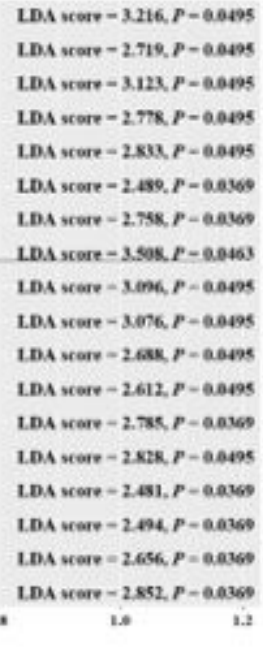

LDA score $-2.719 . P$ - 0.0498 D. scart - 3.12X. P = 0.0695 LD. score -2.889 P -0.099 LDA score $-2.459 . P$ - 0.0360 LDA scate - 3 and, $P$ - 0.045 LDA scere $-2.612, p$ - 0.049 IDA wrate -2.785 - $p$-0.026

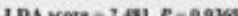

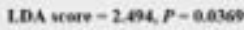

\section{Figure 5}

Differentially abundant microbial taxa displayed by $(A)$ cladogram and (B) bar plot at the genus level affected by endolysin LyJH307 supplementation detected using linear discriminant analysis (LDA) effect size (LEfSe) with a LDF score $\geq 2$. 


\section{(A) KEGG Pathways}

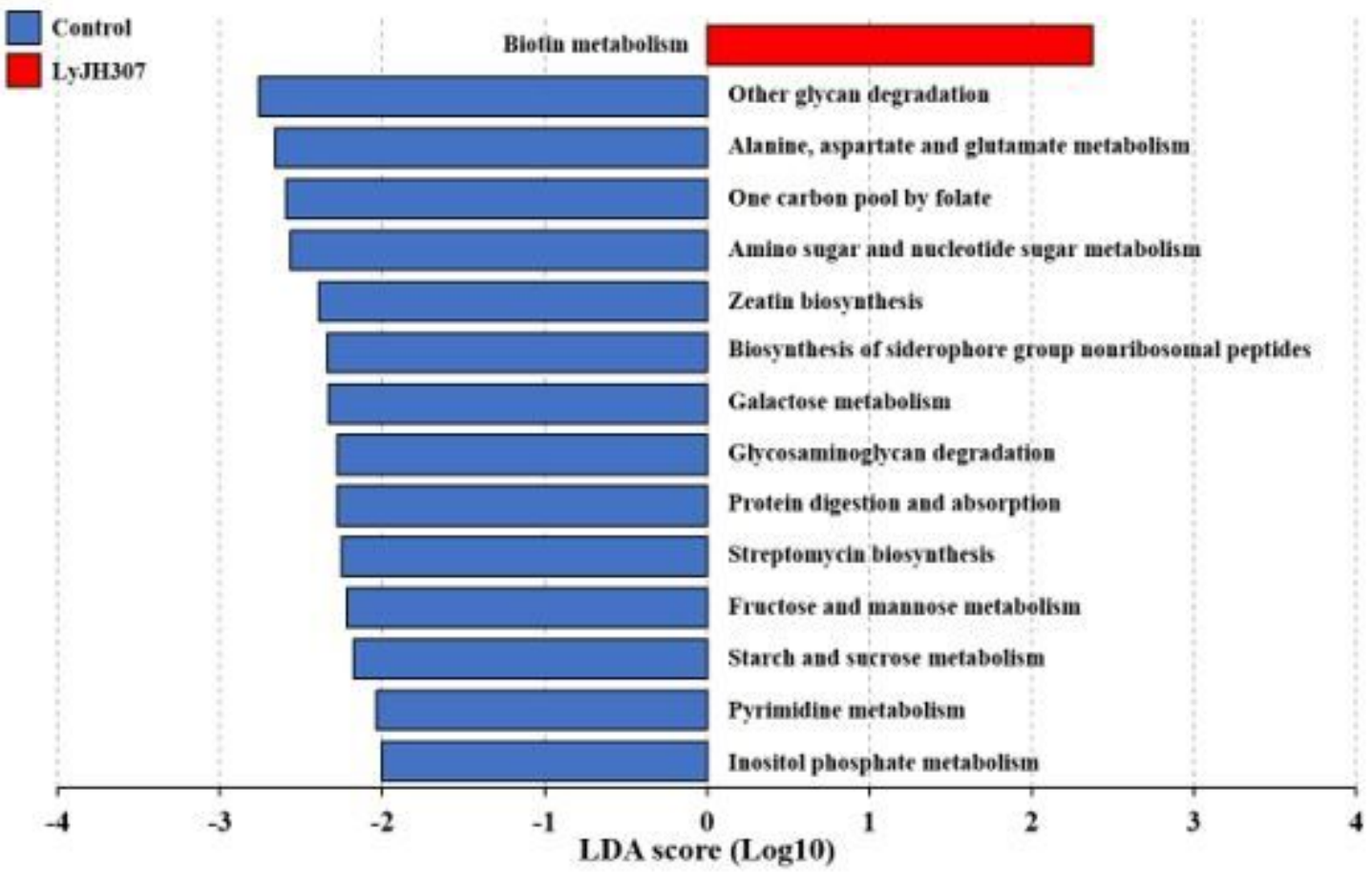

(B) KEGG Modules

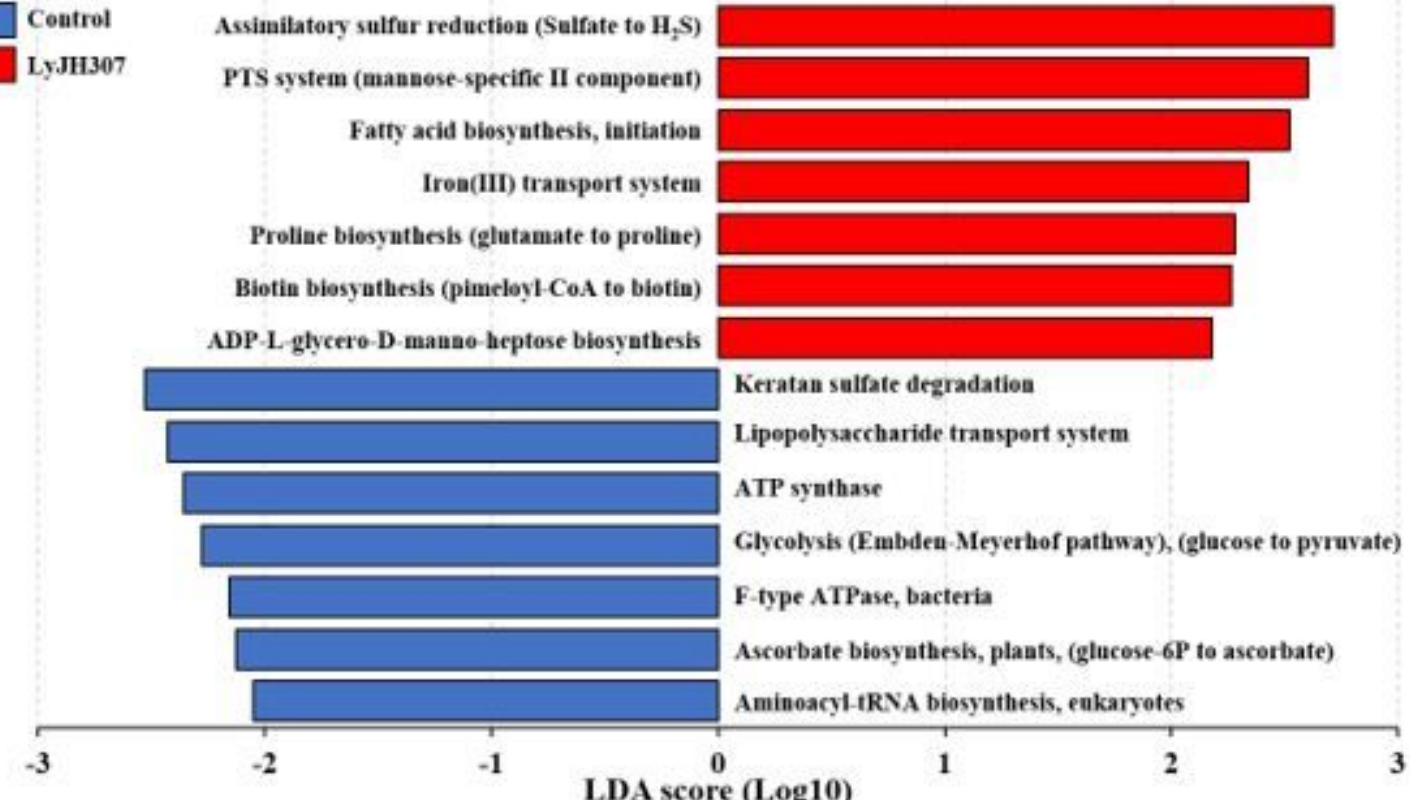

\section{Figure 6}

Differentially abundant predicted functional features of Kyoto Encyclopedia of Genes and Genomes (KEGG) pathways and modules affected by endolysin LyJH307 supplementation detected using linear discriminant analysis effect size (LEfSe) with a linear discriminant analysis effect size $\geq 2$. 


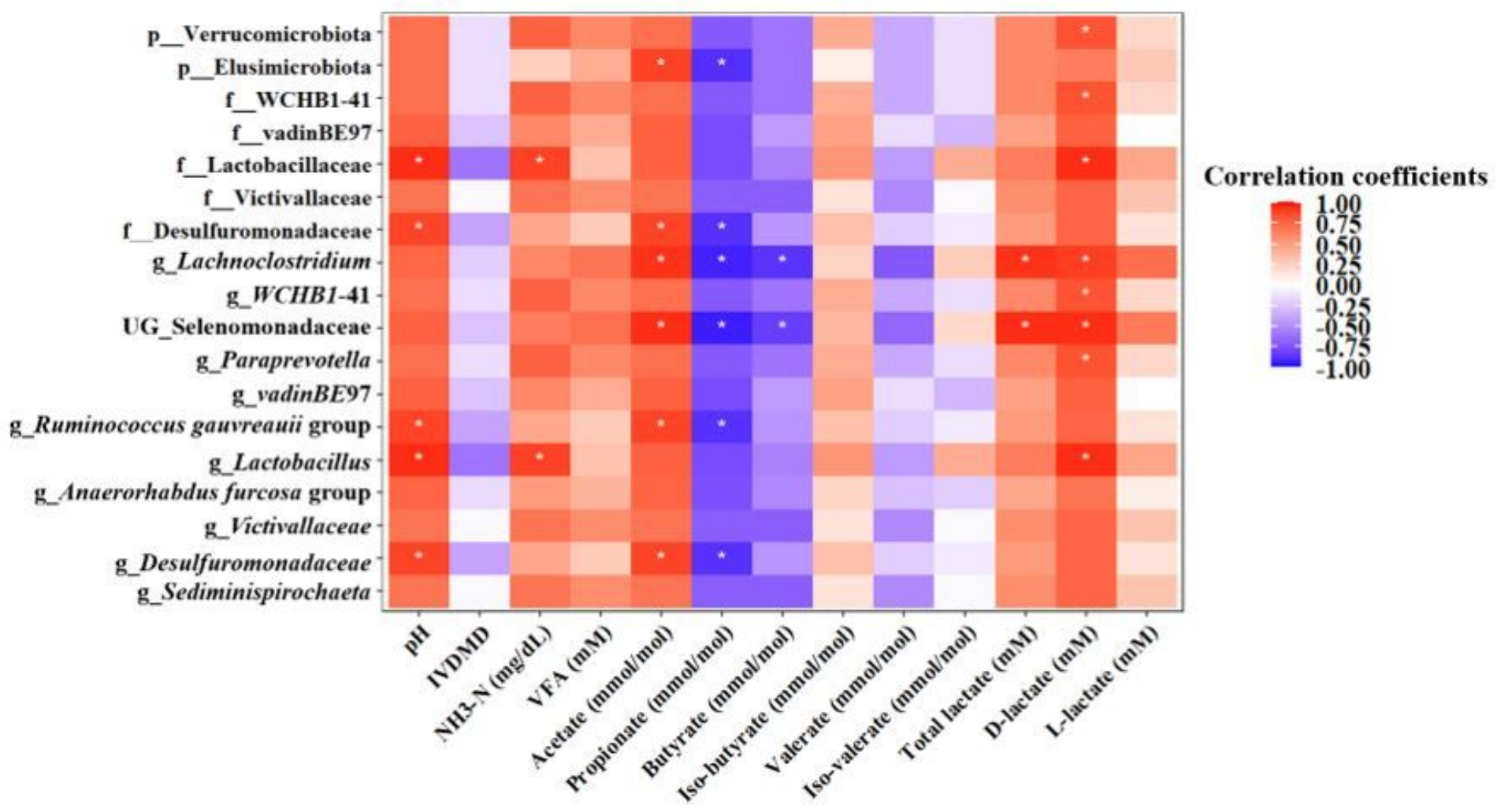

Figure 7

Spearman's correlation coefficients between rumen fermentation characteristics and the relative abundance of differentially abundant prokaryotic taxa at phylum, family, and genus levels by the supplementation of endolysin LyJH307 in an in vitro experiment. Positive correlation (closer to 1) is shown as red, and negative correlation (closer to -1) is shown as blue. Asterisk indicates significant correlations between rumen fermentation characteristics and the relative abundance of taxa $(|r| \geq 0.8$ and $P<0.05)$.

\section{Supplementary Files}

This is a list of supplementary files associated with this preprint. Click to download.

- 210220LyJH307invitrofiguresupplementarytable1.docx 